\title{
High Temperature QCD
}

\section{Maria Paola Lombardo* INFN}

E-mail: lombardo@lnf.infn.it

I review results on QCD at high temperature since lat year lattice conference. Topics include steady progress with staggered fermions and new developments with Wilson type fermions, either in the Quark Gluon Plasma phase, and in the transition region. Simulations with chiral quarks are coming of age, and togheter with theoretical developments trigger interesting developments in the analysis of the critical region. Issues related with the universality class of the chiral transition and the fate of the axial symmetry are discussed in the light of new numericaland analytical results. Transport coefficients and analysis of bottomonium spectra compare well with results of heavy ion collisions at RHIC and LHC. Model field theories, lattice simulations and high temperature systematic expansions help building a coherent picture of high temperature QCD. The (strongly coupled) Quark Gluon Plasma is heavily investigated, and asserts its role of inspiring theoretical laboratory.

The 30th International Symposium on Lattice Field Theory

June 24 - 29, 2012

Cairns, Australia

${ }^{*}$ Speaker. 


\section{From here to high temperature QCD}

High temperature QCD deals with phenomena related with the deconfinement transition towards Quark Gluon Plasma, and the Quark Gluon Plasma phase itself[1].

When heating up matter from room temperature towards temperatures which are best measured in $\mathrm{MeV}$ rather than in ordinary degrees Kelvin we will first produce a gas of the lightest particles - a gas of pions. As temperature becomes hotter a more general gas of resonances, the Hadron Resonance Gas, is though to be a better approximation of the system. Beyond temperatures corresponding to the nucleosynthesis of the lightest nuclei we enter what we can consider a purely hadronic worlds. At a temperature of about $200 \mathrm{MeV}-210^{12}$ Kelvin - hadrons cannot exist anymore, and a transition to the Quark Gluon Plasma takes place. The plasma survives till the temperature of the Electroweak transition, $O(100) \mathrm{GeV}$. All in all, the field of high temperature QCD spans about five orders of magnitude in energy, between the nucleosynthesis of light nuclei up to the Electroweak transition. As we - lattice theorists - know, many of these phenomena, and often the most interesting, take place outside the reach of any analytic approach, making lattice studies mandatory.

This paper describes the developments of this field after last year review [2]. The material fits roughly in two main avenues : one more phenomenological line, which uses $\mathrm{QCD}$, or approximation thereof, to describe mostly the physics of high temperature around the critical region, up to temperatures of about $600 \mathrm{MeV}$. One second line uses models or approximate calculations to learn about general aspects of the same phenomena. This second line reaches up to far higher temperatures, witnessing the deep theoretical interest of the yet largely unexplored physics of the Quark Gluon Plasma.

Before closing this short introduction, one word about the experimental situation. During the first years of operation at RHIC there was a strong focus and motivation to explore the physics of small baryon densities - which, in the Gran Canonical approach we use, corresponds to the physics of $\mu / T<1$., $\mu$ being the quark chemical potential. LHC explores instead near zero density systems, while the search of the elusive endpoint of QCD and the study of other aspects of colder, and denser matter $\mu / T>1$ will be investigated at RUN II at RHIC, and future experiments at FAIR and NICA.

This review will concentrate then on the first aspect - the physics of zero density. The current effort at non-zero baryon density, towards the understanding of the difficult, technical problems still hampering the simulations at large chemical potential, is discussed by Gert Aarts [3].

\section{Thermodynamics : from hadronic Phase to QGP}

The transition from the hadronic phase to the Quark Gluon Plasma is a crossover for physical values of the quark masses. As such, the transition temperature is not uniquely defined and in past years a considerable effort has been devoted to accurate measurements of the pseudocritical temperature identified by different observables. With staggered fermions there is now agreement among different estimates, when the same observables are being used. A key ingredient in resolving this issue has been a careful scale setting procedure. Still not completely converged before Lattice 2012 was the situation of the thermodynamics equation of state, namely the relation be- 

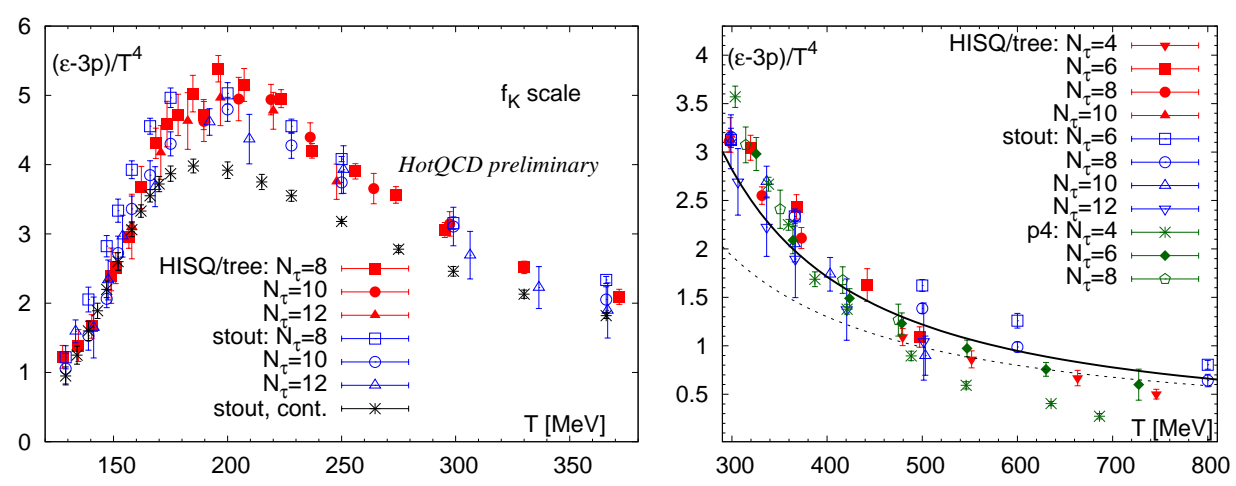

Figure 1: Equation of state for $N_{f}=2+1$ flavours, staggered quarks. The HotQCD collaboration use HISQ/tree action, the Wuppertal-Budapest a stout action. The pion mass in either cases is close to its physical value. Note the different temperature ranges in the two diagrams. (Talk by P. Petreczky)

tween pressure, energy and temperature. The phenomenological relevance is great, since is an input to hydrodynamic calculations [4]. The quantity which is more easily accessible in a lattice calculation is the trace anomaly $\varepsilon-3 P$, often called interaction measure, as it can be computed by differentiating the partition function [5]. It is customary to compare its behaviour to that of the Stefann-Boltzmann limit, corresponding to free fields: convergence to this limit is achieved only at very large temperatures, $T>10^{18} \mathrm{GeV}$, which is taken as one of the evidences of the interacting nature of the Quark Gluon Plasma.

\subsection{Staggered fermions with physical values of $u, d, s$ masses}

Only two collaborations have results with physical values of the up,down, strange mass, in either cases with staggered fermions. In literature and talks prior to Lattice 2012 there are reports of a discrepancy between HotQCD results on the interaction measure $\varepsilon-3 p$ and the WuppertalBudapest stout continuum estimate.

In fig. 1, from Ref.[6] we can see the plot of the trace anomaly $\varepsilon-3 P / T^{4}$ as a function of the physical temperature, as obtained from the two major staggered collaborations using stout [7] and HISQ staggered quarks[6] and physical quark up,down and strange masses. Once the scale is properly set by using $f_{K}$, for a given value of $N_{t}$ the results nicely agree with each other. The extrapolated data - according to the Wuppertal-Budapest collaboration - however lie significantly below the results for a finite $N_{t}$, suggesting residual discretization effects. As thermodynamics is now entering a precision era, perhaps it is useful to fix some significant value of the temperature and compare in details results - similarly to what we would do at $T=0$. I have chosen $T=200$ $\mathrm{MeV}$ - in correspondence to the peak of the interaction measure - and $T=300 \mathrm{MeV}$, see Fig. 2,left. Since for a fixed $N_{t}$ the agreement is very good, I only show the HISQ results here, together with the stout extrapolated results. The relevance of the continuum extrapolation appears clearly in the plots - the extrapolation of the HISQ result depends crucially on which $N_{t}$ one would include. In this sense, the resolution of the residual small discrepancy is narrowed down to the issue of $N_{t}$ 


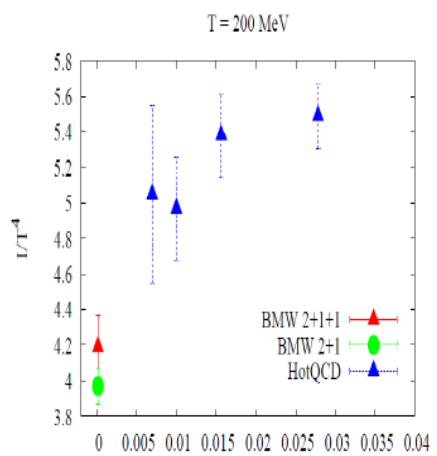

$1 \mathrm{Nt}^{2}$

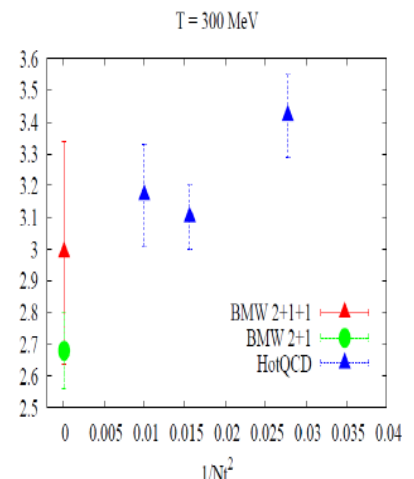

$1 / \mathrm{Nt}^{2}$

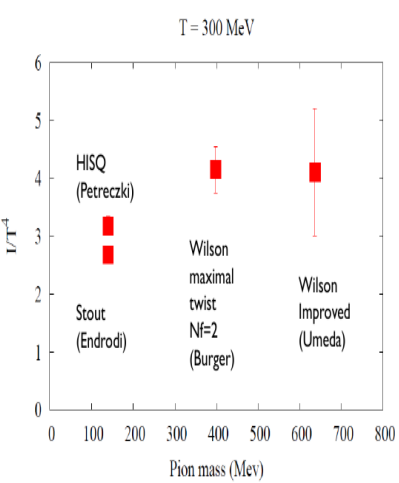

Figure 2: The interaction measure for two selected temperatures as a function of the $1 / N_{t}^{2}$ : results at fixed $N_{t}$ compare favourably, some residual issues with the extrapolation procedure are being settled. On the right, the overall status of the continuum results for $T=300 \mathrm{MeV}$.
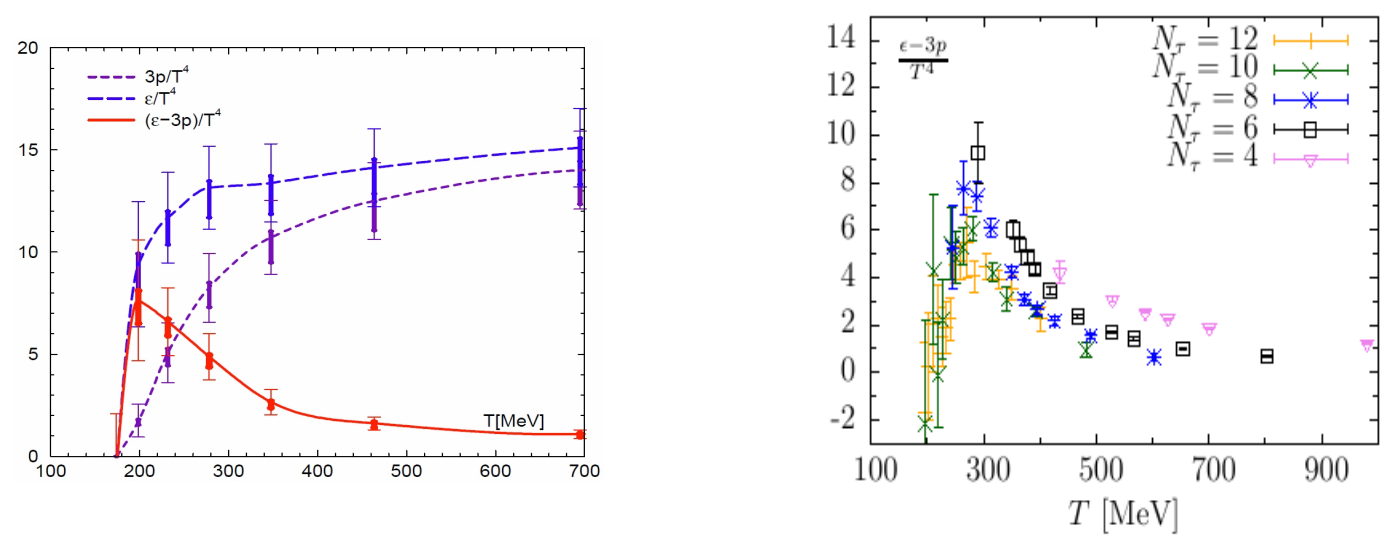

Figure 3: Equation of state for $N_{f}=2+1$ improved Wilson fermions. $m_{\pi} / m_{\rho}=0.63$ (WHotQCD).
Figure 4: Equation of state for $N_{f}=2$ Wilson fermions at maximal twist, $m_{\pi} \simeq 400 \mathrm{MeV}$.(tmft)

extrapolation, and either collaborations are steadily improving their results. The same Fig. 2,right, shows the dependence of bulk thermodynamics on the matter content, to be discussed below.

\subsection{Wilson fermions thermodynamics}

New results for the thermodynamics with Wilson fermions have been presented by the WHOTQCD collaboration[8] and by the tmft collaboration [9]. In either cases the pion mass is larger than $400 \mathrm{MeV}$.

The WHotQCD collaboration presented results for $2+1$ flavors, a physical value of the strange quark mass, and a largish pion mass. Some results are shown in Fig.2.2. The Wilson action was non-perturbatively improved, and the gluonic part used Iwasaki improved glue. Results for two flavors of twisted mass Wilson fermions at maximal twist are available as well, with a pion masses of about $400 \mathrm{MeV}$, and larger4. The extrapolation to the continuum limit seems to be reasonably under control, and the effectiveness of the tree-level correction seems confirmed. 

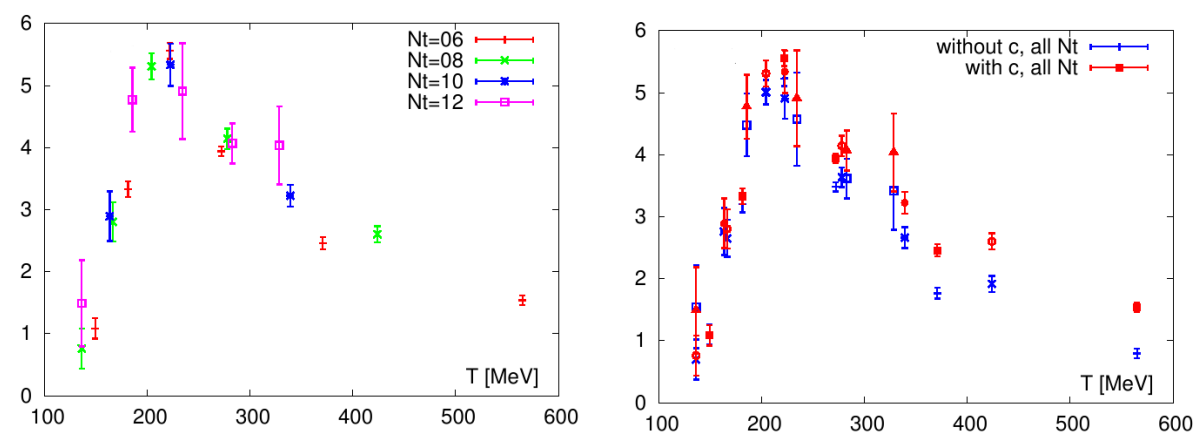

Figure 5: Charm effects on the Equation of State: MILC results for the trace anomaly for $N_{f}=2+1+1$ using HISQ quarks along lines of constant physics. This study was carried out with physical charm and strange mass. The comparison of the two plot allows the identification of the effect of the charm mass on the EoS.

\subsection{Effects of the charm on the EoS}

One step ahead in the matter content was taken by the MILC collaboration and the WuppertalBudapest Collaboration who presented results for $N_{f}=2+1+1$. Contrary to naive expectations, and in agreement with the predictions of effective theories, there is an early onset of the charm contribution at about $T=350 \mathrm{MeV}$ [11]. The MILC collaborations [10] determined the QCD EoS with $2+1+1$ flavors using the HISQ action. Their study was then carried out on lines of constant physics with $m_{l} / m_{s}=1 / 5$, with physical strange and charm quark mass. Above $T_{c}$ the precise value of the light quark mass should not be important. The preliminary data have been obtained with $N_{t}=6,8,10$ and 12, without any attempt at a continuum extrapolation yet. Fig. 5, left shows the MILC preliminary result for the trace anomaly $\varepsilon-3 P$, and in Fig. 5, right, the same is shown but with either without the valence charm quark contribution.

The Wuppertal-Budapest collaboration[12] presented further results on charm effects on the EoS. Their results were also successfully compared with High Temperature perturbation theory, down to $T=350 \mathrm{MeV}$, and with a Hadron Resonance Gas model, which offers a good description of the system up to temperature of about $150 \mathrm{MeV}$. The outcome of this comparison is presented in Fig.6. As discussed at length in previous studies, any simple analysis is expected to break down in the vicinity of the transition, where the critical or pseudocritical behaviour cannot be captured by analytic equation of state. Fig. 6 thus clearly identifies the interesting region where a lattice analysis is mandatory. An overview of the mass sensitivity for $T=300 \mathrm{MeV}$ can be seen in Fig.2, right, where continuum results are shown as function of the pseudoscalar mass.

\section{The transition region - symmetry aspects}

In the limit of massless quarks the QCD Lagrangian enjoys a global chiral symmetry $S U\left(N_{f}\right)_{L} \otimes$ $S U\left(N_{f}\right)_{R} \otimes U_{A}(1) \otimes U_{V}(1)$. The vector $U(1)$ transformation corresponds to baryon number and the axial $U_{A}(1)$ which would promote the symmetry to $U\left(N_{f}\right) \otimes U\left(N_{f}\right)$ is a symmetry of the classical theory but not of the quantum theory. The symmetry breaks down to $S U\left(N_{f}\right) \otimes U_{V}(1)$ at low temperatures. Since up and down quarks, and to some extent the strange quarks, are not too heavy, relics of the critical behaviour can still be observable even in the crossover region for physical 
values of the quark masses - the connection being made within the framework of the magnetic equation of state.

It is speculated that the breaking of the $U_{A}(1)$ symmetry is stronger at zero temperature, or, equivalently, that $U_{A}(1)$ symmetry is effectively restored at high temperature. The fate of the $U_{A}(1)$ effective restoration is closely related with the issue of the order of the chiral transition with two massless flavors, leaving open the possibility of either a first or second order transition depending on the intensity of the residual $U_{A}(1)$ breaking at $T_{c}[15,16]$. In view of the relevance of the symmetries, it is obviously extremely desirable to have fermionic formulations which respect chiral symmetry. Remember now that staggered and Wilson fermions break the $U_{A}(1)$ symmetry, and that chiral symmetry is also

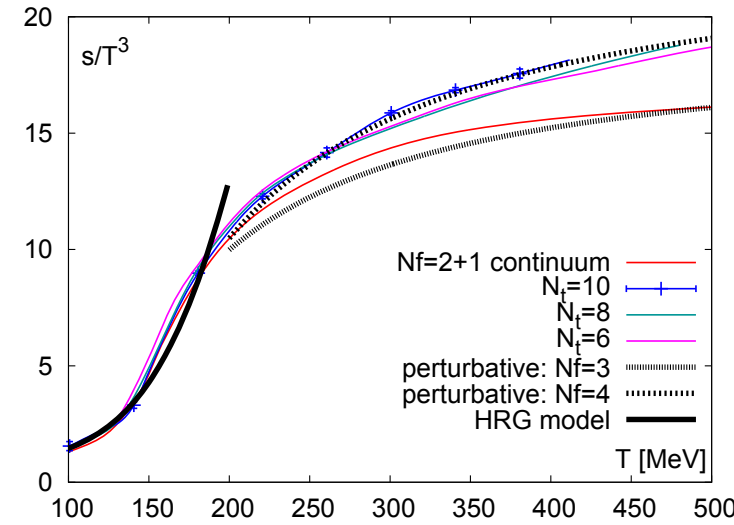

Figure 6: The entropy for the $\mathrm{Nf}=2+1+1$ flavors from the Wuppertal-Budapest group:the low temperature results compare well with the HRG model, while high temperature results demonstrate the sensitivity to the charm in the perturbatively predicted region broken in the Wilson formulation, while an $U(1)$ symmetry (unrelated with the $U_{A}(1)$ symmetry) is preserved by staggered fermions, which however do not allow a local definition of $U_{A}(1)$ symmetry. The computationally expensive overlap quarks have an exact chiral symmetry. Domain wall fermions approximate it with a precision which is in principle arbitrary, the residual chiral symmetry breaking being characterised by an additive renormalisation to the quark mass $m_{\text {res }}$ which vanishes in the limit of infinite 5-th dimension: $m_{\text {res }}\left(L_{S}\right)=c_{1} \frac{e^{-\lambda L_{s}}}{L_{s}}+c_{2} \frac{1}{L_{s}}$.

New results with Wilson fermions with $2+1$ and 2 flavors, as well as results with chiral fermions were presented at this conference, with emphasis on two interrelated open issues: the fate of the $U_{A}(1)$ symmetry at the phase transition, and the universality class for $N_{f}=2$.

\section{$3.1 U_{A}(1)$}

The physics of the anomaly is particularly intriguing, given its role in the universality class of the transition $[15,16]$. Moreover, should the anomaly not restored at the transition, one would have the opportunity to disentangle its effects from those of chiral symmetry.

Patterns of symmetries are reflected in the interplay of scalars susceptibilities, according to the well known MILC plot [13,14]. The order parameter for $S U(2) \otimes S U(2)$ is related to the longitudinal susceptibility $\langle\bar{\psi} \psi\rangle / m=\chi_{\pi}$, and a candidate order parameter for $U_{A}(1)$ is $\chi_{\pi}-\chi_{\sigma}$. It is however true in general that if an observable $\mathscr{O}$ is not invariant under a given symmetry $\mathscr{S}$, $<\mathscr{O}\rangle=0$ is a necessary, but not sufficient condition for $\mathscr{S}$ to be exact. This means that the candidate order parameter for the $U_{A}(1)$ symmetry $\chi_{\pi}-\chi_{\sigma}$ might well be zero even if $U_{A}(1)$ is still broken.

Chiral observables are related to the low-lying eigenmodes of Dirac operator:

$$
<\bar{\psi} \psi>=\int d \lambda \rho(\lambda) \frac{2 m}{m^{2}+\lambda^{2}}
$$



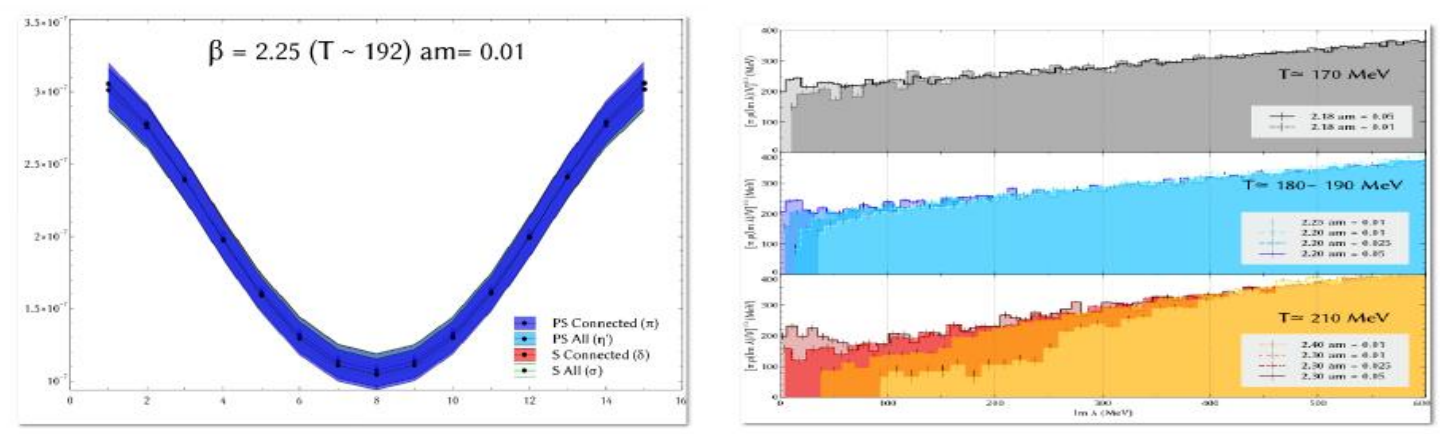

Figure 7: Direct observation of the degeneracy in the scalar sector, right above $T_{c}$, left. The eigenvalue distribution for several temperatures, hinting at the restoration of the $U_{A}(1)$ symmetry. Overlap fermions, two flavors, JLQCD

$$
\chi_{\pi}-\chi_{\delta}=\int d \lambda \rho(\lambda) \frac{4 m^{2}}{\left(m^{2}+\lambda^{2}\right)^{2}}
$$

so the fate of the $U_{A}(1)$ symmetry can be read off the shape of the eigenvalue density $\rho(\lambda)$. The various possibilities are extensively discussed discussed in a recent paper by the HotQCD Collaboration [21].

A new theoretical analysis has been presented by Aoki[17, 18] and collaborators, who find

$$
\lim _{m \rightarrow 0}<\rho^{A}(\lambda)>_{m}=\lim _{m \rightarrow 0}<\rho_{3}^{A}>_{m} \frac{\lambda^{3}}{3 !}+O\left(\lambda^{4}\right)
$$

i.e. no constant contribution to the spectrum, which implies at high temperature

$$
\lim _{m \rightarrow 0}\left(\chi_{\pi}-\chi_{\sigma}\right)=0
$$

when $\left\langle\bar{\psi} \psi>=0\right.$. In other words, for any $N_{f}$ two point correlators would be blind to the breaking of $U_{A}(1)$ when chiral symmetry is restored. Note that older results (refs. [19, 20]) reached a similar conclusion on the non-observability of $U_{A}(1)$ non restoration in two point functions, but only for $N_{f} \geq 3$. The new analysis [17] requires a careful consideration of the order of the limits and suggests that early work was inaccurate.

Results presented by JLQCD[22], with two flavors of overlap fermions and physical values of the pion mass, apparently realise the scenario proposed by Aoki[17] indicating the degeneracy of all the scalar correlators in the chiral limit above $T_{c}$, which is of course a stronger statement than the degeneracy of the susceptibilities, see Fig. 7, left. Moreover, a gap in the eigenvalues' distribution opens up right above $T_{c}$, as shown in Fig. 7, right. The conclusion still needs to be corroborated to a better control of systematics.

The same issue was investigated with domain wall fermions. New results with $2+1$ flavors were presented at this meeting[23] on the $32^{3} \times 8$ lattices by RBC/LLNL, and ensembles with physical quark masses on the $32^{3} \times 8$ and $64^{3} \times 8$ are being planned from HotQCD. The behaviour of chiral susceptibilities which can be seen in Fig.8, left top, is on the overall convincing, with small deviations from the existing staggered results. Fig.8,left bottom, shows the ratio of dimensionless ratios at $\beta=1.75$, demonstrating violation of the order of only $5 \%$ of an ideal scaling. The spectral 

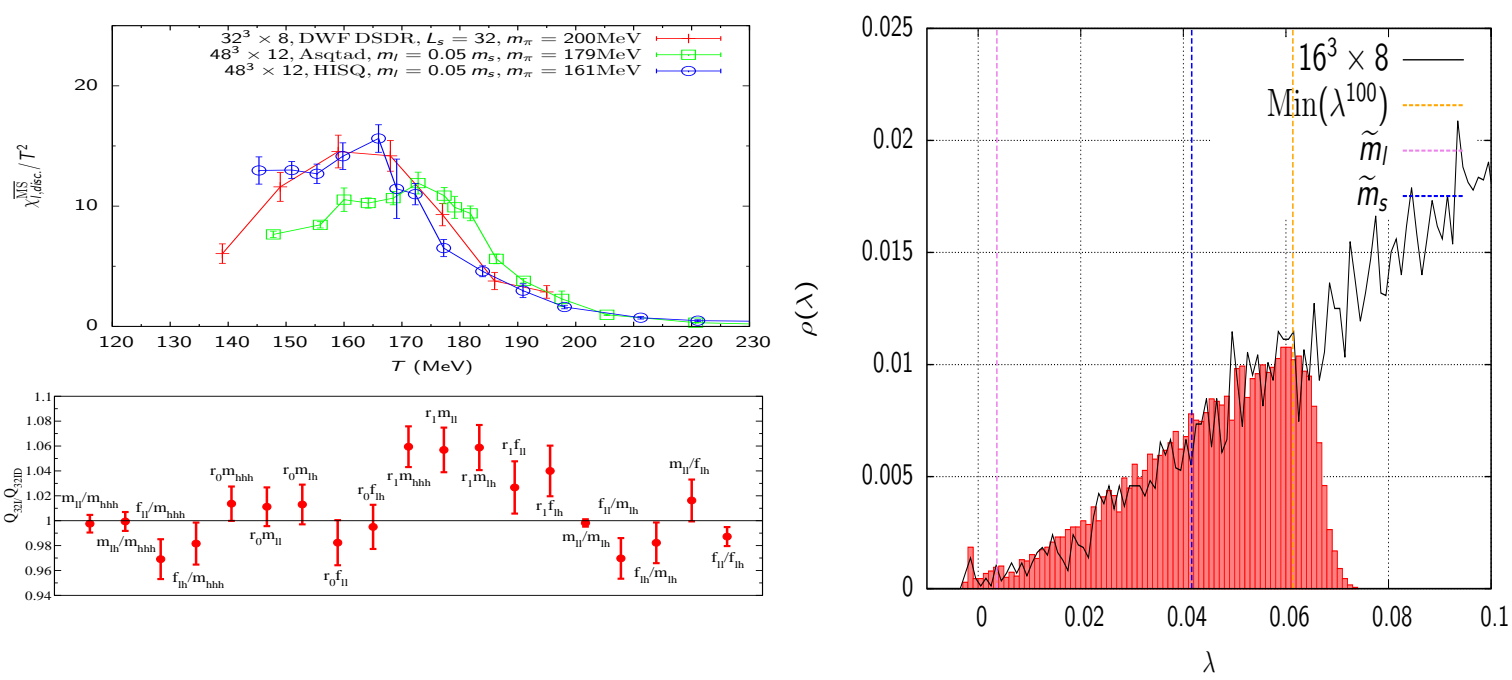

Figure 8: Domain wall fermions via-a-vis staggered results(top left). The scale independence for domain wall fermions (bottom left). The eigenvalue spectrum (right) favors a non restored $U_{A}(1)$ symmetry beyond the QCD transition, however the largish masses and the residual breaking leave room for a different conclusion (Talk by J. Lin, HotQCD and RBC/LLNL).

densities are shown in Fig.8, right, for a temperature above, but close to $T_{c}$. The distribution shows a clear peak near zero ("near zero modes") and a linear behavior extending down to zero. This may correspond in the continuum limit to $\rho(\lambda)=c_{1} \delta(\lambda)+c_{2} \lambda$. at a variance with Aoki's scenario, and implying a residual $U_{A}(1)$ breaking with $\chi_{\pi}-\chi_{\delta} \neq 0$ in the chiral limit.

All these analysis require an extrapolation to the chiral limit, hence studies with different masses - which are in progress - are needed to draw definite conclusions. However a detailed study with HISQ fermions[24], 2+ 1 flavors, pion masses ranging from $115 \mathrm{MeV}$ till $230 \mathrm{MeV}$ leave the conclusion open, with a detailed discussion of the systematic effects affecting a proper analysis of the eigenvalue shape.The results for the distributions are presented in Fig. 3.2. Even at the highest temperatures there is no evidence of a gap around zero. More than any comment the shape of the eigenvalues clearly demonstrate the uncertainties affecting the conclusions.

\subsection{Further studies around the critical temperature}

Further studies with chiral fermions were presented by the TWQCD Collaboration, using two flavors of Domain Wall fermions [25] and by the Wuppertal-Budapest collaboration[12, 26], who use two flavors of overlap fermions, with topology fixing a la JLQCD. These studies can be regarded as still preparatory since the pion masses exceed $300 \mathrm{MeV}$, and the analysis concentrates on basic observables. The Wuppertal-Budapest collaboration 10 presented also a comparison with continuum staggered results which is at first sight satisfactory[12, 26]. The transition region was also studied with Wilson fermions, $2+1$ flavors, including continuum extrapolation[27, 28]. The pion masses range from 125 to $275 \mathrm{MeV}$, and studies with smaller masses are in progress. Again the focus is on the agreement with staggered quarks, see Fig.3.2, which so far looks very nice, within the largish errors. New results for two flavors of non-perturbatively $O(a)$ improved Wilson 

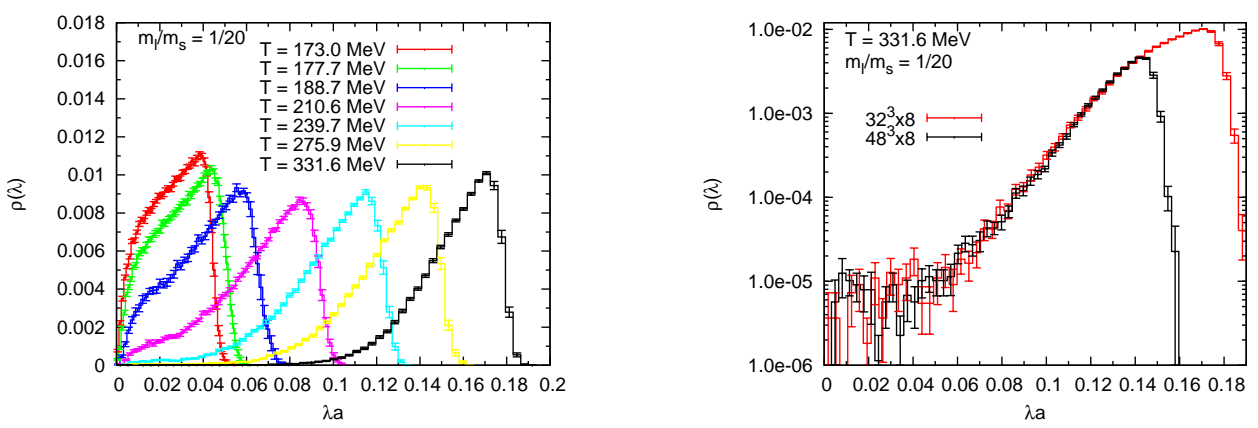

Figure 9: Eigenvalue distribution from HISQ fermions: the shape determines the fate of $U_{A}(1)$ symmetry which remains open. The left diagram shows temperature dependence of the Dirac spectrum at temperatures in the range $173.0-331.6 \mathrm{MeV}>T_{c}$. The right diagram shows the volume dependence of spectral density at the highest temperature. The volume dependence is quite small and there remains a tail even at this temperature. (Talk by H. Ohno)
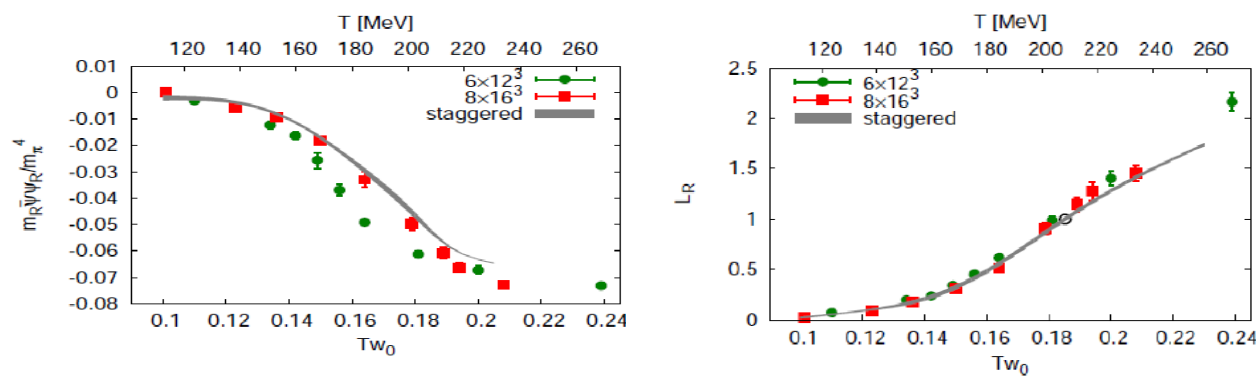

Figure 10: The crossover region with overlap fermions, $N_{f}=2, m_{\pi}=350 \mathrm{MeV}$, from the Wuppertal Budapest collaboration
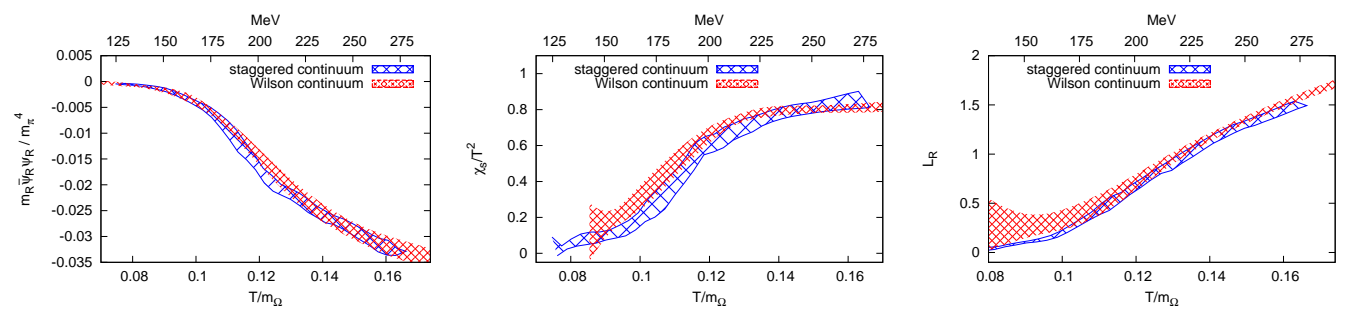

Figure 11: The renormalised chiral condensate, renormalised Polyakov loop and strange quark susceptibility from the Wuppertal-Budapest simulations with 2+1 flavors of Wilson fermions (Talk by D. Nogradi) 

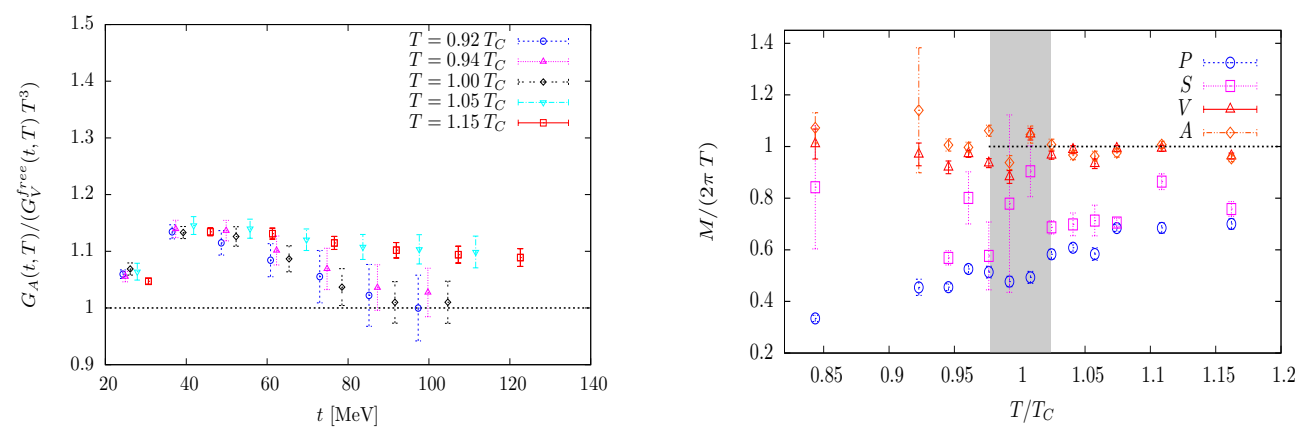

Figure 12: Vector Correlators in the plasma, from simulations with two flavors of improved Wilson fermions on large lattices (left), and summary of results (right).(Talk by B. Brandt)

fermions have been presented [60] working along lines of constant physics with $m_{\pi} \simeq 290 \mathrm{MeV}$. The picture, Fig. 12, shows the axial vector correlator normalised to the free vector correlators. The right-side picture shows the collection of screening masses themselves - the degeneracy in the vector sector which anticipates the one in the scalar sector might suggest a persistence of $U_{A}(1)$ breaking, although it could also be that the near degeneracy in the vector sector comes from the largish masses values.

\subsection{The universality class for $N_{f}=2$}

As noted above, the order of the transition for two flavor QCD is a dynamical issue which depends on the magnitude of the residual breaking of $U_{A}(1)$ at the transition point. The situation can be depicted as in Fig. 13, left: the order might well change along the transition line even if the symmetries are the same on both sides. Since quarks chemical potentials do not alter the chiral symmetries in the Lagrangian one can study a possible realisation of this scenario by varying $\mu$, away from the condensation phases, and see whether one can observe first and second order transitions. This strategy is pursued $[29,30,31]$ with imaginary chemical potential. Fig. 14 shows the

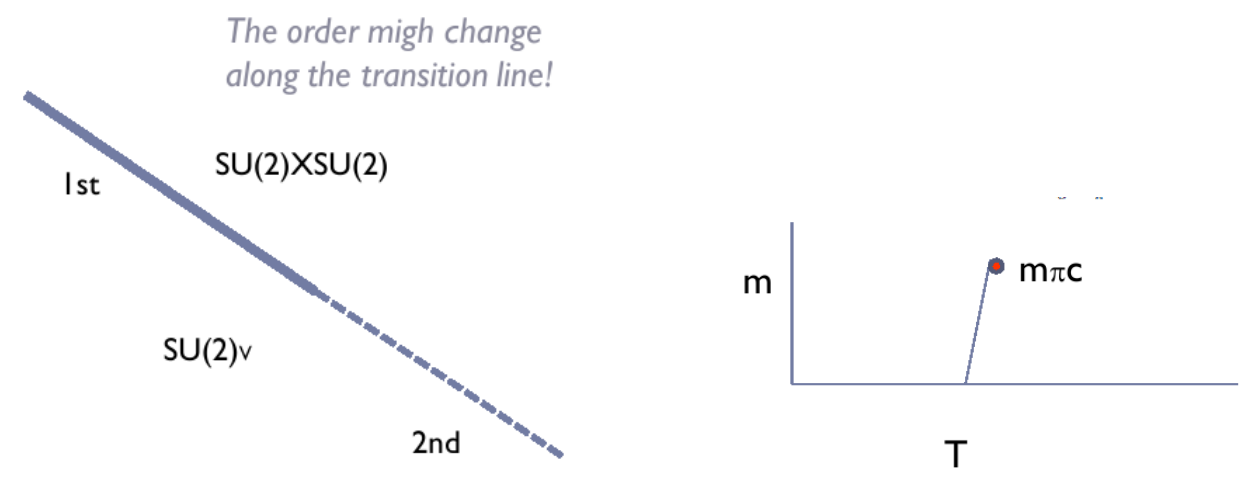

Figure 13: Sketchy view of a possible behaviour of the transition line: the order might change along the line even when the symmetries of the two phases are the same(left); the region of the first order transition is characterised by the endpoint at finite mass, in the universality class of the $Z_{2}$ Ising model 

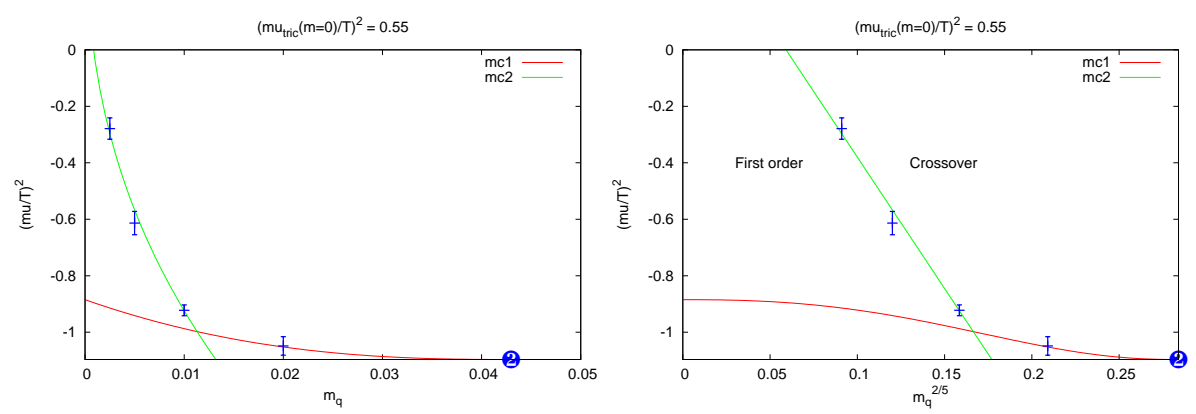

Figure 14: Search for universality class for the $\mathrm{Nf}=2$ theory : the position of the endpoint of the first order transition for imaginary $\mu$ versus the bare mass. The transition becomes of second order when the endpoint hits zero, and based on these plots the authors suggest that this might happen for a positive $\mu^{2}$. The results have been obtained for $N_{t}=4$ unimproved staggered fermions hence they are far away from the continuum (Communicated by Ph de Forcrand)

results with imaginary baryonic $\mu$ [29], and Fig.15 shows the results for imaginary isospin $\mu$ [30]. The authors argue that following the fate of the endpoint of the first order transition, sketched in Fig. 13, right, is a practical way to decide the order of the transition at $\mu=0$. In particular one might want to locate the turning point between a first and a second order transition: since first order transitions were observed for a non-zero imaginary chemical potential for $N_{f}=2$, a turning point for real $\mu$ implies that the phase transition at zero chemical potential is of first order[29, 30]. Current extrapolations suggest that this is realised for $N_{t}=4$, for unimproved staggered fermions, hence far from the continuum limit.

The transition for two flavor close to the continuum limit is being investigated with Wilson fermions [9], and some results are shown in Fig. 16. The plot depicts the current situation with Wilson fermions, maximal twist and improved gauge sector. The extrapolation to the chiral limit can be carried out either with a second order ansatz, or assuming a critical endpoint smaller than $300 \mathrm{MeV}$. The data do not discriminate between these two possibilities. This implies that the order of the $N_{f}=2$ transition in the massless continuum limit is still undecided, and suggests that the endpoint of the first order transition at $\mu=0$, if any, is for $m_{\pi}<300 \mathrm{MeV}$.
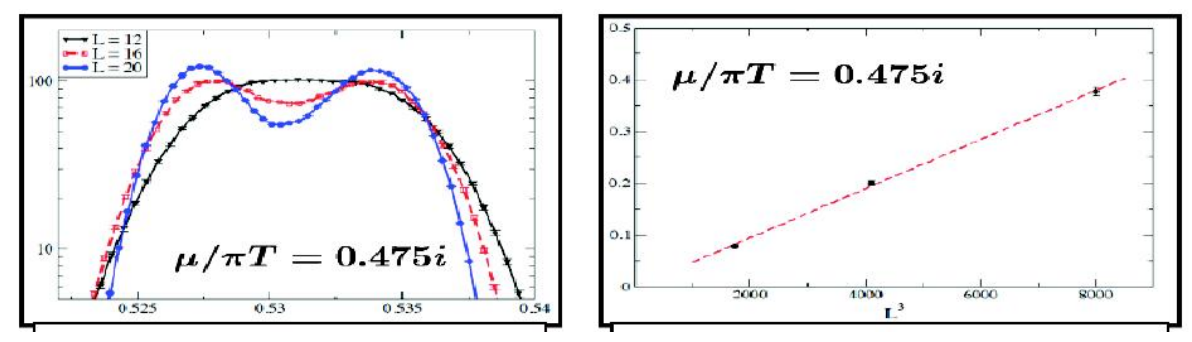

Figure 15: Direct observation of a first order transition at non-zero imaginary isospin density. If this signal would persist till zero chemical potential the $N_{f}=2$ transition would be of first order (Talk by D. Cosmai) 


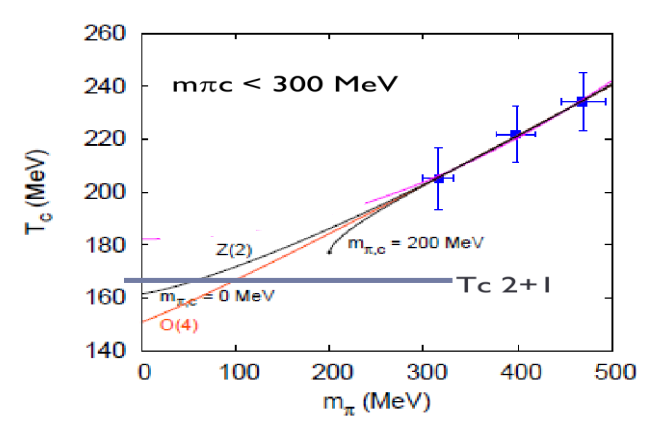

Figure 16: Results on the crossover temperature for $N_{f}=2$, with chiral extrapolation as motivated by the plausible universality classes (Talk by F. Burger, tmft)

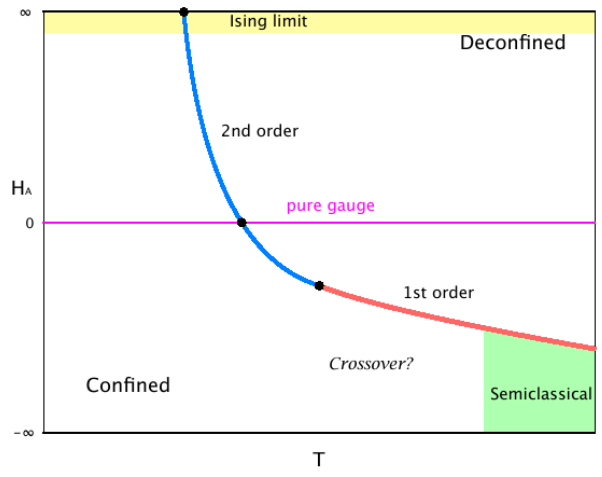

Figure 17: The phase diagram in an enlarged parameter space (Talk by Ogilvie)

\section{The transition region - confinement, gauge dynamics, external fields, $\theta$ term}

The discussions in the previous Section focused on the fermionic sector of the theory, and the associated chiral symmetries. The "obligatory" step at this point is to remind ourselves that gauge dynamics and confinement are crucial, yet mysterious, aspects of the transition, in particular due the lack of a bona-fide order parameter for deconfinement.

Abelian gauge theories are an important exception : in these theories the confinement transition is understood as a condensation of topological defects - in the case of a four dimensional $U(1)$, for instance, strings of magnetic monopoles [32].

Ogilvie proposes to search for a continuous path between non-abelian theories and abelian ones by suitably enlarging the parameter space, [33]. The considered phase diagram is depicted in Fig. 17. By supplementing the $S U(2)$ action with a double trace term, one can show that the confined phase of $S U(2)$ and the high temperature confined phase of the expanded model are continuously connected; the latter is amenable to a semiclassical treatment and in turn is continuously connected to the confined phase of the abelian model.

A more direct interpretation of the transition in terms of magnetic monopoles has been recently proposed by Shuryak [34]. In Fig.18 lattice data for different $N_{f}$ are used to estimate the strength of the coupling at the transition, which is then compared with the coupling at the IRFP[36, 37]. In particular the data marked with a diamond were obtained with the same action,and different number of flavor, and can be meaningfully considered together [35]. The coupling at the transition becomes stronger and stronger with increasing $N_{f}$ : the proposed explanation is that light fermions can occupy the (chromo)magnetic monopoles, making them unsuitable for Bose Einstein condensation. Lattice simulations measuring magnetic monopole properties can confirm this proposal, which, if true, would lend support to confinement scenario based on monopole condensation in QCD. A further related observation is the emergence of the conformal window of QCD for large number of flavors - reviewed by Joel Giedt[38] - as the zero temperature limit of a super-strongly interactive Quark Gluon Plasma.

It has been proposed that fluctuation of topological charge might play a role at the transition, producing measurable $P$ and $C P$ violations in heavy ion collisions. In the Lagrangian $\mathrm{P}$ 

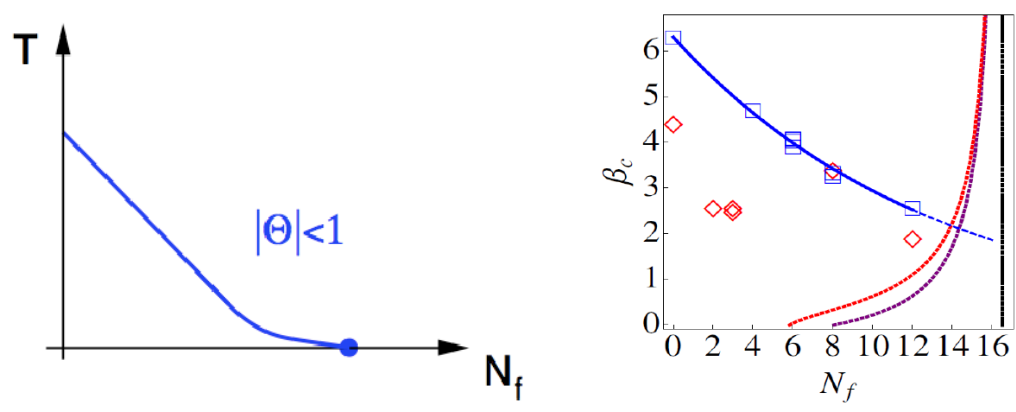

Figure 18: The transition as a function of matter content (left) and the associated coupling according to Shuryak. Results from various lattice groups
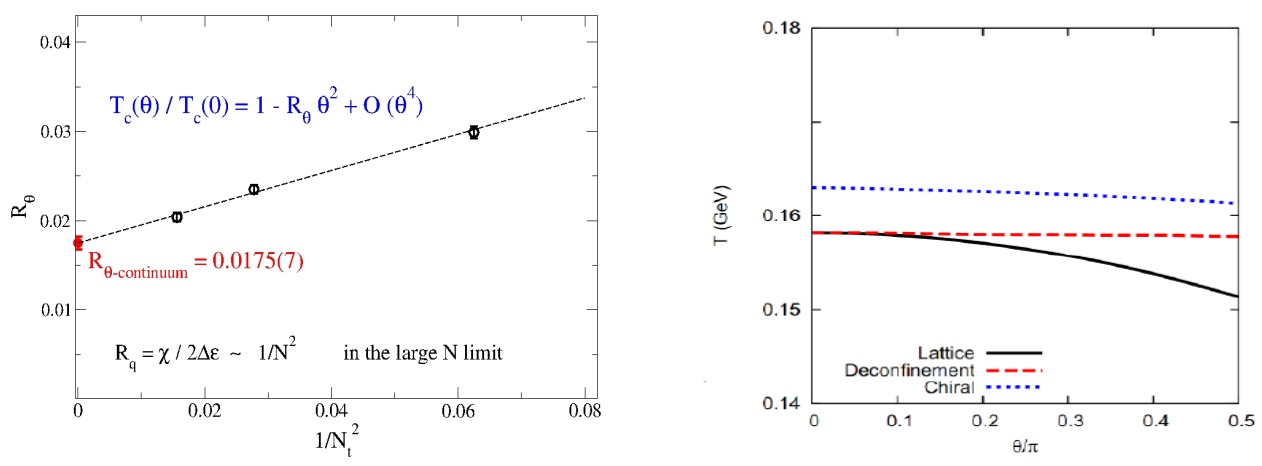

Figure 19: News on the $\theta$ vacuum: the continuum extrapolation of the slope of the critical temperature from lattice simulations at imaginary $\theta$ (left, talk by F. Negro), and from model studies(right, talk by S.Sasaki).

and CP violating terms enter via the $\theta$ term $\theta Q(x), Q(x)$ being the topological charge density. Despite the experimental bound $\theta<10^{-10}$ it is still conceivable that such term affects the critical temperature. New studies on the $\theta$-dependence of the deconfinement temperature have been presented[39, 40, 41], the former numerical and the latter based on a model analysis. In both cases $T(\theta)$ was parametrised as $T(\theta)=T_{c}-R_{\theta} \theta^{2}$, and the coefficient of the quadratic coefficient $R_{\theta}$ has been estimated. The results are shown in Fig.19. Both studies agree on the sign of $R_{\theta}$ term, as well as on its very little magnitude, with a substantial agreement within the largish systematic errors.

Finally, an interesting line of research is the effect of an external field on the phase transition[42]. As magnetic fields as large as $10^{14}$ Tesla can be produced at the QCD transition a lattice effort has started to compute the effects of strong magnetic fields on the QCD transition, and on the magnetic catalysis - a possible enhancement of chiral symmetry due to the effects of the external fields. Although intuitively an external magnetic fields should favour chiral breaking, model analysis give non-univocal results, and, again, lattice calculations are mandatory to settle the issue. I refer the interested reader to a recent review[43]. Details of the dynamics are still under discussion, particularly close to the chiral limit. Recent results have been obtained for two [44] and three colours[45]. 


\section{Fluctuations}

Lattice QCD simulations are being carried out in a GranCanonical ensemble. This allows fluctuations of conserved quantities - baryon number, electric charge, strangeness - for instance, the baryon number should be zero on the average, but it can fluctuate configurations by configurations. In ordinary conditions fluctuations are quite small: for instance one can compute the distribution of baryon number in ChPT and find that it is described by a narrow Gaussian [46, 47] while when the temperature increases the distribution broadens and might change shape[46, 47]. The fluctuations thus reflect properties of the thermal medium and of the critical point[48].

In general, a synthetic way to describe a distribution is via its momenta

$$
\mu_{r}=<X^{r}>=\int_{-\infty}^{+\infty} X^{r} f(X) d X
$$

The momentum generating function $M(\xi)=<\exp (\xi X)>=\sum_{r=0}^{\infty} \frac{\xi^{r}}{r !}<X^{r}>$ is a simple way to combine momenta into a single expression and we recognise that the momenta are linked with the coefficients of the Taylor expansion of the generating functional $\left.<X^{r}\right\rangle=\left[\frac{d^{r}}{d \xi^{r}} M(\xi)\right]_{\xi=0}$. Consider then the pressure $p=T / V \ln \mathscr{Z}(\mathscr{T}, \mu)$ and its Taylor expansion in powers of $\mu / T$ around $\mu=0$ :

$$
\frac{p(\mu / T)}{T^{4}}=\sum_{n=0}^{\infty} c_{n}(T)\left(\frac{\mu}{T}\right)^{n}
$$

and we recognise that the zero density fluctuations of the quark number density $n(\mu)=\frac{\partial p(T, \mu)}{\partial \mu}$ are given by the coefficients $c_{n}(T)$. Similar reasoning holds for the other conserved charges. Results for the momenta have been successfully compared with Hadron Resonance Gas till temperatures very close to $T_{c}[49,50]$.

At the time of the meeting new analytic results from A. Vuorinen and collaborators [51] became available, followed by a publication, [52]. The excellent agreement between analytic and numerical results - which can be appreciated in Fig.20 - is in part due to the elimination of the pure gauge component (by taking the derivatives w.r.t to $\mu$.) Note also in the same figure the very good agreement between the results of the Wuppertal-Budapest collaboration and HotQCD: these measurements have been performed on the same set of gauge fields discussed above and the agreement here again corroborate the optimistic view that the residual minor disagreements are almost solved. A completely different approach to the dynamics of fluctuations is offered by the flux tube model presented by Patel[53]: the idea is that the fluctuations should be 'visible' in the gauge fields, and one can conceive, within the flux tube models, one way to visualise the gauge field dynamics in position space. The 'rules of the game' in a first approximation are simple: quark and anti-quark are bond by a string in a $\bar{Q} Q$ state. The string can break via the formation of a light-heavy meson, $\bar{Q} Q \rightarrow q \bar{Q}+\bar{q} Q$, or in the plasma as $\bar{Q} Q \rightarrow \bar{Q} q+q q Q$, see Fig 21. In this way one might hope to study multi-correlation particles which are useful for phenomenology, and highlight the microscopic structure of the medium, for instance by distinguishing a dilute liquid from a cristal-like structure. 


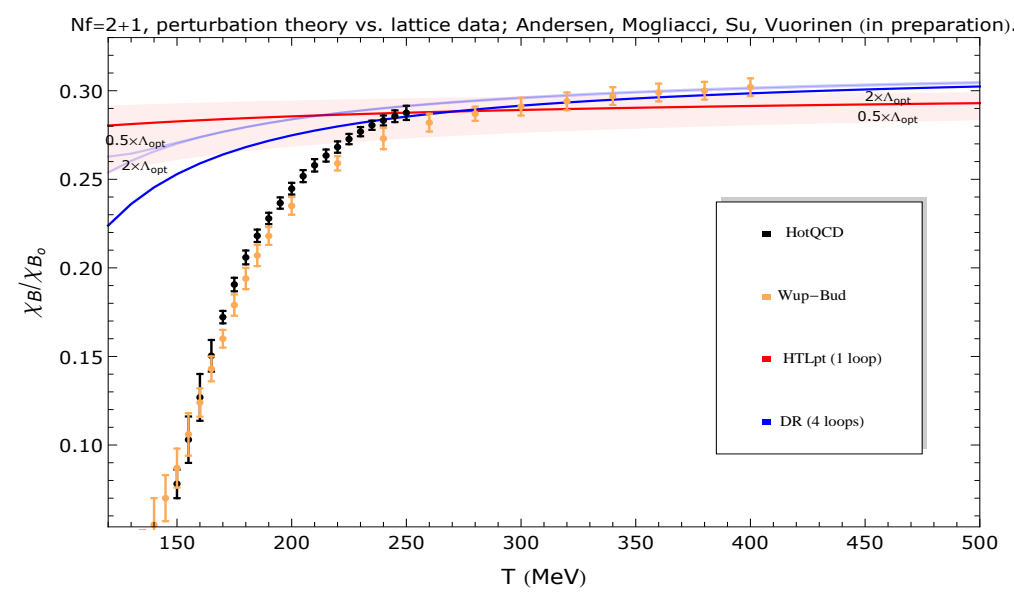

Figure 20: Lattice results for the baryonic susceptibility vis-a-vis analytic studies. Note the agreement between different lattice calculations, and with the 4 loops dimensionally reduced theory

\section{Quark Gluon Plasma - a new world}

The physics of the Quark Gluon Plasma is of course addressed by most of the studies described before. Here -somewhat arbitrarily - I collect those studies which are motivated by the desire of a deeper and general theoretical understanding of the physics of this unusual state, without reference to specific models.

One popular way to learn about general features is to enlarge the parameter space of interest: a large number of colours, or a large number of flavors. For a large number of colours $N_{c}-$ reviewed by M. Panero[54] - we have one very interesting finite temperature result[55]. Panero and collaborators have studied the behaviour of the Polyakov loop in the deconfined phase of a $S U\left(N_{c}\right)$ Yang-Mills theory. Different representations and different $N_{c}$ exhibit a near universal behaviour once trivial scaling factors are taken into account, see Fig. 22. This observation - so far restricted

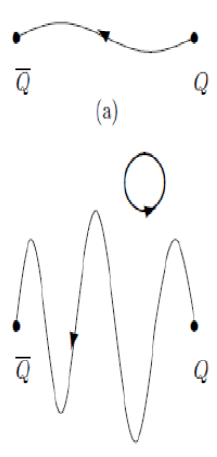

(b)
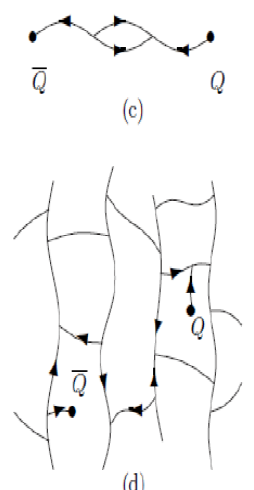

$\mathrm{T}=\mid 460 \mathrm{MeV}$

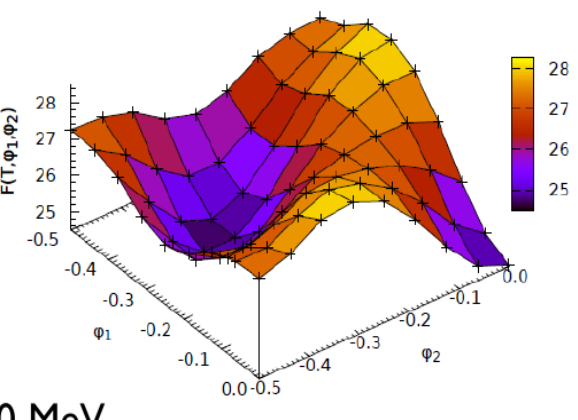

Figure 21: Describing fluctuations: the flux tube model by Patel (left)-The Polyakov loop analysis by Gattringer (right). 

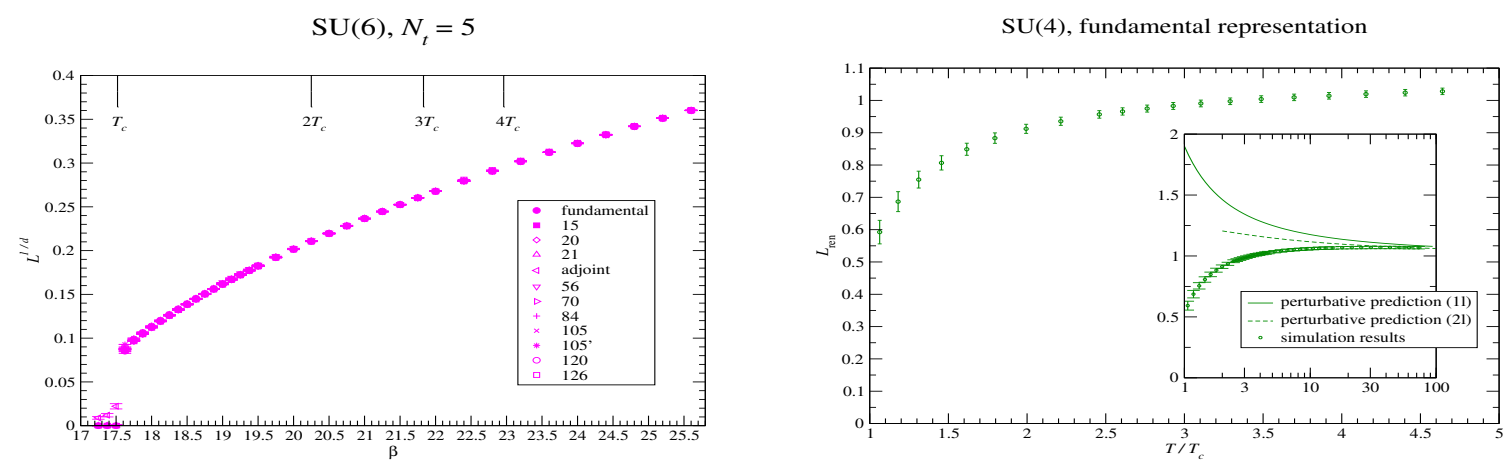

Figure 22: The left diagram shows the bare loop for $S U(6)$, and different representations, rescaled assuming Casimir scaling-the universality of the results support the assumption. The right-hand diagram shows the same renormalised loop in the fundamental representation for $S U(4)$-in both cases the behaviour is very similar to the one observed in $S U(3)$. (M.Panero et al.)

to pure gauge systems - lends support to large $N$ approaches to the analysis of the high temperature phase of QCD.

It is believed that as chiral symmetry is no longer spontaneously broken, the only remaining scale in the massless model is the temperature. Would the eigenvalue spectrum of QCD give information on any remaining scale? Kovacs[56] has shown that this is indeed the case - the equivalent of the Thouless energy was found to scale as the temperature itself.

Attempts at modelling the Quark Gluon Plasma by use of Polyakov loop dynamics has produced the observation that $Z_{3}$ metastable states might become important at $T>750 \mathrm{MeV}$ [57]. Such metastable states with dynamical fermions might have an influence on bubble formation and on the system's dynamics in general.

How to detect directly the dynamics of the Polyakov loop? Diakonov, Gattringer and Schadler [58] propose a free energy for the untraced Polyakov loop $X$

$$
F(T, X)=T \int_{0}^{\beta} d \beta^{\prime}<S>_{T, X, \beta^{\prime}}
$$

where $X$ is defined by

$$
P=\frac{1}{V_{3}} \sum_{t=1}^{N_{t}} U_{4}(\vec{x}, t)=\operatorname{Tr} X
$$

and

$$
<S>_{T, X, \beta^{\prime}}=-\frac{\partial}{\partial \beta^{\prime}} \ln Z\left(T, X, \beta^{\prime}\right)
$$

This definition, so far tested in the pure Yang Mills case, compares very well with high $T$ perturbation theory and open the way to a rigorous approach to the Polyakov loop dynamics.

\section{Quark Gluon Plasma confronts experiments}

How far we can go with comparing our results with experiments? For orientation, we know that during $\mathrm{Au}-\mathrm{Au}$ collisions at $200 \mathrm{GeV}$ at $\mathrm{RHIC}$ the system reached up temperatures between $340-380 \mathrm{MeV}$, the current LHC runs $-2.76 \mathrm{TeV}$ - explored up to $420-480 \mathrm{MeV}$ with possibly hot 
spots of about 500-600 MeV. LHC at full luminosity will reach about $1 \mathrm{GeV}-\operatorname{six}$ times $T_{c}$ [59]. The recent CMS results[61] are a good starting point for the discussion: the invariant mass plot measured in pp shows the expected three bumps in correspondence to the fundamental $\Upsilon$ state and the first two radial excitations. When the same distribution is being measured by colliding lead ions, the lowest peak is almost stable, while the two higher peaks have almost disappeared. A natural thing to do is to compare these observations with the heavy quark spectrum computed on the lattice - a notoriously difficult task. see eg. Ref [62]for a recent comprehensive overview.

In early times a lattice computed potential was used as input for the Schroedinger equation for heavy mesons. Later on, issues were risen concerning the appropriate potential to use, and most important it was finally realised that the correct potential should include an imaginary component to account for the broadening of the state[63, 64] Alternative calculations of the potential using the wave function as an input to the Schroedinger equation, and a reverse engineering to extract the potential itself have been presented at this meeting as well[66], and the results can be seen in Fig. 7.

At the other extreme - w.r.t quantum mechanical, non-relativistic studies - we have field theoretic, relativistic calculations of the spectral functions:the euclidean charmonium correlator 7 is computed[67] - in this case on quenched configurations -, and the spectral function is then reconstructed by use of by now standard Maximum Entropy Method analysis (MEM)[68].

All these approaches give results broadly consistent with each other, and with intuition. How-

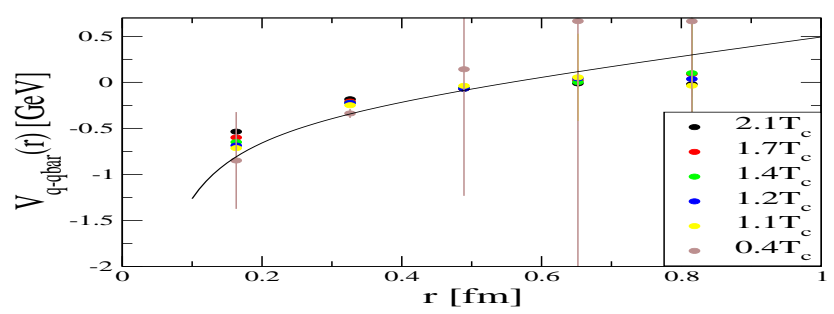

Figure 23: A new strategy for the potential analysis: Interquark potential obtained by reverse engineering the Schroedinger equation after calculations of the wavefunction. (Talk by Ch. Allton)
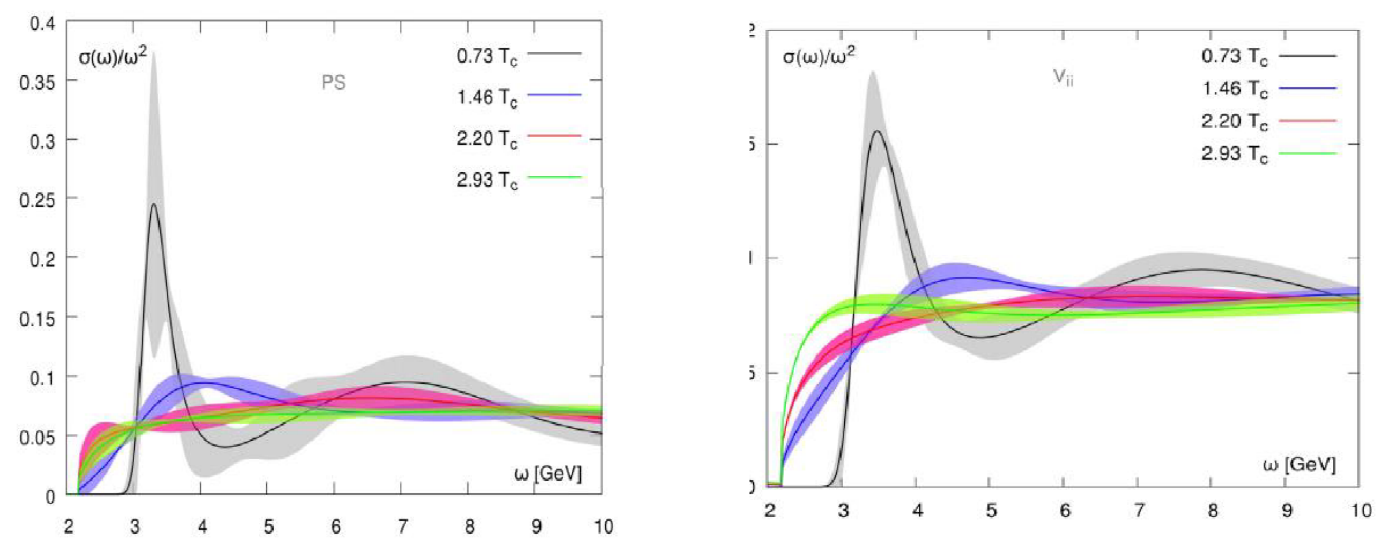

Figure 24: Charmonium spectral functions from a full relativistic calculation[67] 

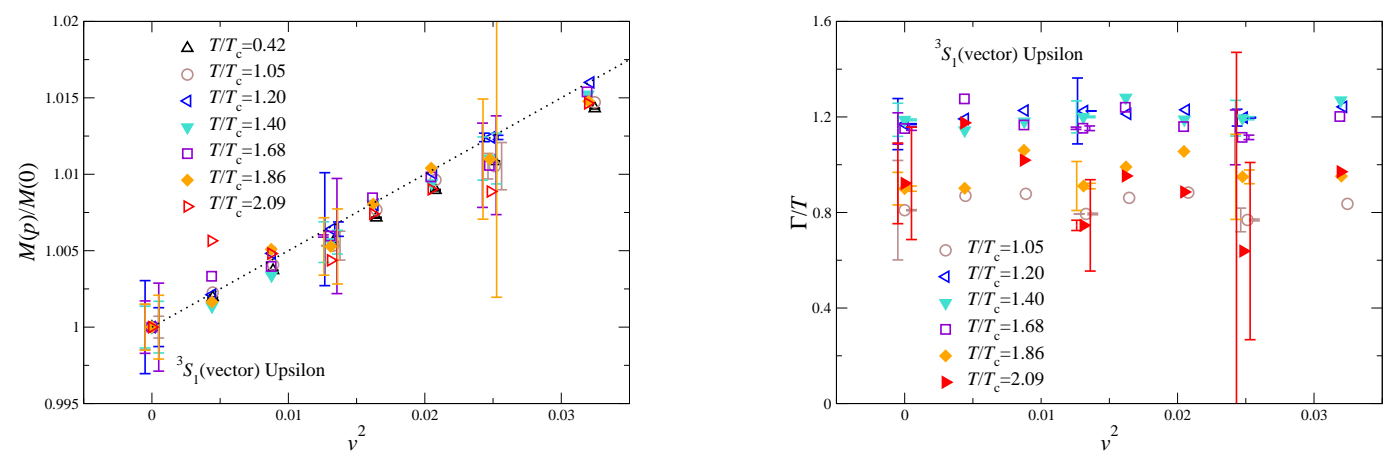

Figure 25: Momentum dependence of spectral functions in the plasma, as seen in the mass and width. Position of the ground state peak $M(p v) / M(0)$ (left) and the upper limit on the width of the ground state peak, normalized by the temperature, $\Gamma / T$ (right), as a function of the velocity squared $\left(v^{2}\right)$ in the vector $(\Upsilon)$ channel [70]

ever a solid understanding of the systematics is still lacking.

An improvement to the implementation of the Maximum Entropy Method has been presented by A. Rothkopf [65]. The method proposes to use an extended search space, and preliminary successful results have been obtained by use of mock data: the numerical behaviour is more stable, and the resolution is satisfactory.

One further approach consists of evaluating NRQCD propagators over dynamical gauge fields: the reconstruction of the spectral functions is made easier[69] as in this case the spectral relation reduces to

$$
G(\tau)=\int_{-2 M}^{\infty} \frac{d \omega^{\prime}}{\pi} \exp \left(-\omega^{\prime} \tau\right) \rho\left(\omega^{\prime}\right),
$$

As a result, the spectral function is merely the inverse Laplace transform of the correlator, and all problems associated with thermal boundary conditions are absent. The new NRQCD results[70] presented this year include a systematic study of momentum dependence, as well as a study of the mass dependence[71]. Successful comparison with the predictions of effective models has started, together with a more detailed analysis of the systematics.

Directly related with the computation of spectral functions are the transport coefficients, see e.g Ref. [72] for a comprehensive review. New results appeared this year concerned heavy quark diffusion. In the framework of a Gaussian and Markovian Langevin dynamics, the diffusion constant $D$ describes the mean square displacement for times $t$ larger that the relaxation time $<|\Delta(x)|^{2}>=6 D t$. In the strong coupling limit of the SUSY Yang-Mills plasma one finds, using holographic duality

$$
D=\frac{2}{\pi T} \frac{1}{\sqrt{\lambda}}
$$

Gubser[73] suggests $\lambda \simeq 6 \pi$ in QCD, concluding $D \simeq 1 /(2 \pi T)$. In a relativistic setup, transport coefficients are defined as

$$
\lim _{\omega \rightarrow 0} \frac{\rho(\omega}{\omega^{2}}
$$




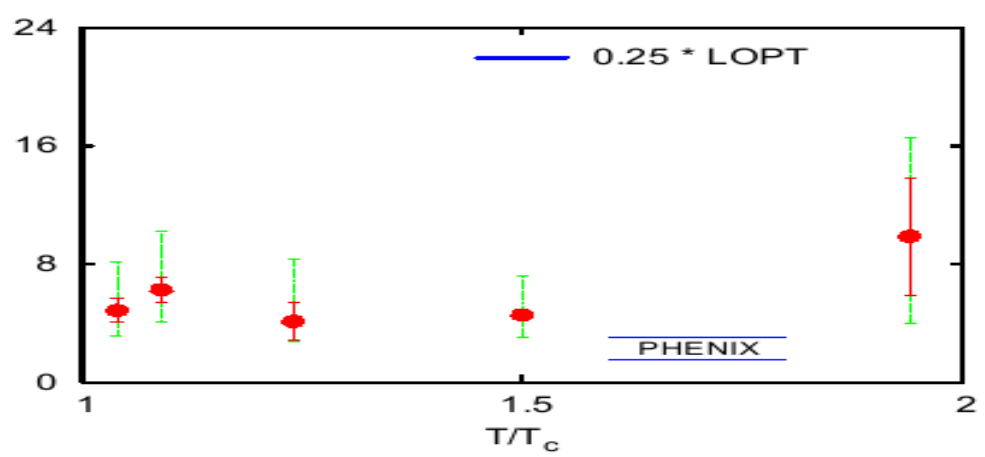

Figure 26: Heavy quarks diffusion coefficients : impressive agreement with experimental results, corroborating a strongly interactive quark gluon plasma - the limiting case is shown as a short segment on the right (Talk by S. Datta).

where $\rho(\omega)$ is a spectral function in the appropriate channel[74]. For instance $D$ has been extracted from the charmonium spectral functions described above[67], with results remarkably close to the one obtained in strongly coupled SUSY, eq.7.2.

The diffusion coefficient $D$ can also be extracted by pure gauge correlators[75]: results for this have been presented by Datta[76]. Again the result is close to the one of eq.7.2, hence in remarkable agreement with those from Ref.[67].

The comparison with experimental results is shown in Fig.26. Needless to say, it will be of a great interest to see the new results from LHC, at higher energies, when the plasma is expected to be less bound, and the transport coefficient should correspondingly increase.

To appreciate the quality of the lattice results one should keep in mind that the LOPT results, see again Fig. 26 - to be achieved in the extremely high temperature limit - would be

$$
D=\frac{6 \pi}{g^{4} T \log \left(2 T / m_{D}\right)} \simeq 80
$$

On the scale of the huge range of allowed $D$ values, from the extreme strongly coupled to the free plasma, the agreement between the two lattice calculations, carried out with different strategies and actions, is impressive.

\section{Summary}

A precision era for high temperature QCD has started, with results whose accuracy in many cases is comparable with the one achieved at zero temperature. Results for bulk thermodynamics in the continuum and at the physical point for staggered results well into the current LHC region are in good control, and residual discrepancies seem minor. Wilson fermions are becoming competitive, with results in the continuum limit (albeit still with largish masses) for bulk thermodynamics, and in the critical region. New analytic studies in the high temperature phase compare remarkably well with numerical results, down to unexpectedly low temperatures. Chiral fermions are coming of age, with new, continuously developing high quality results for domain wall and overlap fermions. Good chiral properties together with new theoretical insight trigger further activity on chiral and 
axial symmetries and their spectral properties. Details of QCD dynamics prove to be extremely important and delicate close to the phase transitions: the fate of the axial anomaly, the order of the transition for $\mathrm{Nf}=2$, the response to a magnetic field, and to $\theta$ therm are still unclear. New lattice results for transport and quarkonia have been presented which compare well with RHIC and LHC experiments. The (strongly coupled) Quark Gluon Plasma is heavily investigated, either in QCD and by use of deformed QCD and other model field theories, and confirms its role of inspiring theoretical laboratory.

\section{Acknowledgements}

I am most grateful to the many colleagues who have shared their results and notes. I have prepared this talk during a visit in Bielefeld in Summer 2012, were I have greatly enjoyed, and benefited from many discussions, and in particular I wish to thank Edwin Laermann, York Schroeder, Alexi Vuorinen and Christian Schmidt. Finally, it is a pleasure to thank the organisers for a very interesting and smoothly run meeting.

\section{References}

[1] B. V. Jacak and B. Muller, Science 337 (2012) 310

[2] L. Levkova, PoS LATTICE 2011 (2011) 011

[3] G.Aarts, these Proceedings

[4] J. Casalderrey-Solana, H. Liu, D. Mateos, K. Rajagopal and U. A. Wiedemann, Gauge/String Duality, Hot QCD and Heavy Ion Collisions, arXiv:1101.0618 [hep-th]

[5] see e.g. C. DeTar and U. M. Heller, Eur. Phys. J. A 41 (2009) 405; O. Philipsen, The QCD equation of state from the lattice, arXiv:1207.5999 [hep-lat].

[6] P. Petreczky [HotQCD], these Proceedings

[7] S. .Borsanyi, G. Endrodi, Z. Fodor, S. D. Katz, S. Krieg, C. Ratti and K. K. Szabo, JHEP 1208 (2012) 053

[8] T. Umeda [WHotQCD], these Proceedings

[9] F. Burger[tmft], these Proceedings

[10] U. Heller [MILC], these Proceedings

[11] M. Laine and Y. Schroder, Phys. Rev. D 73 (2006) 085009

[12] S. Krieg [Wuppertal-Budapest], these Proceedings

[13] E. V. Shuryak, Comments Nucl. Part. Phys. 21 (1994) 235

[14] C. W. Bernard, T. Blum, C. E. Detar, S. A. Gottlieb, U. M. Heller, J. E. Hetrick, K. Rummukainen and R. Sugar et al., Phys. Rev. Lett. 78 (1997) 598

[15] R. D. Pisarski and F. Wilczek, Phys. Rev. D 29 (1984) 333

[16] A. Butti, A. Pelissetto and E. Vicari, JHEP 0308 (2003) 029

[17] S.Aoki, these Proceedings

[18] S. Aoki, H. Fukaya and Y. Taniguchi, arXiv:1209.2061 [hep-lat] 
[19] S. H. Lee and T. Hatsuda, Phys. Rev. D 54 (1996) 1871

[20] T. D. Cohen and X. -D. Ji, Phys. Rev. D 55, 6870 (1997)

[21] A. Bazavov et al. [HotQCD Collaboration], Phys. Rev. D 86 (2012) 094503

[22] G. Cossu [JLQCD], these Proceedings

[23] J. Lin [RGB/HotQCD], these Proceedings

[24] H. Ohno, these Proceedings

[25] H. Hsieh, these Proceedings

[26] S. Borsanyi, Y. Delgado, S. Durr, Z. Fodor, S. D. Katz, S. Krieg, T. Lippert and D. Nogradi et al., Phys. Lett. B 713 (2012) 342

[27] D.Nogradi, these Proceedings

[28] S. Borsanyi, S. Durr, Z. Fodor, C. Hoelbling, S. D. Katz, S. Krieg, D. Nogradi and K. K. Szabo et al., JHEP 1208 (2012) 126

[29] C.Bonati,M.D’Elia, Ph. de Forcrand, O. Philipsen, F. Sanfilippo, in progress at the time of Lat2012, communicated by $\mathrm{Ph}$. de Forcrand

[30] D.Cosmai, these Proceedings

[31] P. Cea, L. Cosmai, M. D’Elia, A. Papa and F. Sanfilippo, Phys. Rev. D 85 (2012) 094512

[32] A.M. Polyakov, Gauge Fields and Strings

[33] M.Ogilvie, these Proceedings

[34] J. Liao and E. Shuryak, Phys. Rev. Lett. 109 (2012) 152001

[35] K. Miura, M. P. Lombardo and E. Pallante, Phys. Lett. B 710 (2012) 676

[36] D. Schaich, these Proceedings

[37] T. Nunes da Silva, these Proceedings

[38] J.Giedt, these Proceedings

[39] F. Negro, these Proceedings

[40] M. D’Elia and F. Negro, Phys. Rev. Lett. 109 (2012) 072001

[41] C. Sasaki, these Proceedings

[42] D. E. Kharzeev, K. Landsteiner, A. Schmitt and H. -U. Yee, arXiv:1211.6245 [hep-ph].

[43] M. D'Elia, Lattice QCD Simulations in External Background Fields, arXiv:1209.0374 [hep-lat]

[44] E. -M. Ilgenfritz, M. Kalinowski, M. Muller-Preussker, B. Petersson and A. Schreiber, Phys. Rev. D 85, 114504 (2012)

[45] G. S. Bali, F. Bruckmann, G. Endrodi, Z. Fodor, S. D. Katz and A. Schafer, Phys. Rev. D 86 (2012) 071502

[46] M. P. Lombardo, K. Splittorff and J. J. M. Verbaarschot, Phys. Rev. D 81 (2010) 045012

[47] M. P. Lombardo, K. Splittorff and J. J. M. Verbaarschot, Phys. Rev. D 80 (2009) 054509

[48] M. A. Stephanov, Phys. Rev. Lett. 102 (2009) 032301 
[49] A. Bazavov et al. [HotQCD Collaboration], Phys. Rev. D 86 (2012) 034509

[50] S. Borsanyi, Z. Fodor, S. D. Katz, S. Krieg, C. Ratti and K. Szabo, JHEP 1201 (2012) 138

[51] Andersen, Mogliacci, Su, Vuorinen, in preparation at the time of Lattice 2012, private communication with A. Vuorinen and S. Mogliacci.

[52] J. O. Andersen, S. Mogliacci, N. Su and A. Vuorinen,

[53] A. Patel, these Proceedings

[54] M. Panero, these Proceedings

[55] A. Mykkanen, M. Panero and K. Rummukainen, JHEP 1205 (2012) 069

[56] T. Kovacs, these Proceedings

[57] M. Deka, S. Digal and A. P. Mishra, Phys. Rev. D 85 (2012) 114505

[58] D. Diakonov, C. Gattringer and H. -P. Schadler, JHEP 1208 (2012) 128

[59] I wish to thank Ulli Heinz for correspondence on this point

[60] B. Brandt, these Proceedings

[61] S. Chatrchyan et al. [CMS Collaboration], arXiv:1208.2826 [nucl-ex].

[62] O. Kaczmarek, arXiv:1208.4075 [hep-lat]

[63] N. Brambilla, J. Ghiglieri, A. Vairo and P. Petreczky, Phys. Rev. D 78 (2008) 014017

[64] A. Rothkopf, T. Hatsuda and S. Sasaki, Phys. Rev. Lett. 108 (2012) 162001

[65] A. Rothkopf, these Proceedings

[66] C. Allton, these Proceedings

[67] H. T. Ding, A. Francis, O. Kaczmarek, F. Karsch, H. Satz and W. Soeldner, Phys. Rev. D 86 (2012) 014509

[68] M. Asakawa, T. Hatsuda, Y. Nakahara, Prog. Part. Nucl. Phys. 46 (2001) 459-508

[69] G. Aarts, C. Allton, S. Kim, M. P. Lombardo, M. B. Oktay, S. M. Ryan, D. K. Sinclair and J. I. Skullerud, JHEP 1111 (2011) 103

[70] G. Aarts, C. Allton, S. Kim, M. P. Lombardo, M. B. Oktay, S. M. Ryan, D. K. Sinclair and J. -I. Skullerud, arXiv:1210.2903 [hep-lat].

[71] S. Kim, these Proceedings

[72] A. Adams, L. D. Carr, T. Schaefer, P. Steinberg and J. E. Thomas, New J. Phys. 14 (2012) 115009

[73] S. S. Gubser, Phys. Rev. D 76 (2007) 126003

[74] H. B. Meyer, Eur. Phys. J. A 47 (2011) 86

[75] D. Banerjee, S. Datta, R. Gavai and P. Majumdar, Phys. Rev. D 85 (2012) 014510

[76] S. Datta, these Proceedings 